\title{
ELASTIC FORM FACTORS IN POINT-FORM APPROACH
}

\author{
MARCO RADICI \\ Dipartimento di Fisica Nucleare e Teorica, Università di Pavia \\ and Istituto Nazionale di Fisica Nucleare, Sezione di Pavia, I-27100 Pavia, Italy
}

\begin{abstract}
Using the point-form approach to relativistic quantum mechanics, a covariant framework is presented for the calculation of proton and neutron electromagnetic form factors. Results for charge radii, magnetic moments, and electric as well as magnetic form factors are produced using the wave functions obtained in the socalled Goldstone-boson-exchange constituent quark model. All the predictions are found in remarkable agreement with existing experimental data.
\end{abstract}

\section{Introduction}

The key issue for a QCD-motivated description of low-energy strong interactions is the identification of the effective degrees of freedom that describe the hadron phenomena. By considering the features of the baryon spectrum, the well-known spontaneous breaking of chiral symmetry suggests that below a certain energy threshold the effective "particles" to be considered should be the constituent quarks, whose dynamical mass is related to the $\langle q \bar{q}\rangle$ condensate, and the Goldstone bosons arising from the dynamical symmetry breaking and coupling directly to the constituent quarks.

The Constituent Quark Model (CQM) based on the assumption that the quark-quark interaction is mediated by the Goldstone Boson Exchange (GBE) 1 , is capable of giving a consistent description of the low-energy spectra of light and strange baryons $\mathrm{B}$. It assumes a linear confinement, as suggested by lattice QCD, and retains only the spin-spin component of the pseudoscalar GBE hyperfine interaction.

However, the CQM should also provide a comprehensive description of other hadron phenomena. As a first test of the model wave functions, here we consider the properties of the nucleon that can be explored with an electromagnetic probe: charge radii, magnetic moments and elastic form factors. In order to get a reliable test, the uncertainties introduced in the calculation of the scattering amplitude need to be minimized. To this aim, a proper treatment of the relativistic kinematics is required. The suggested value of the mass parameter for the constituent quark indicates that traditional nonrelativistic expansions in $p / m$ are not justified 3 . Moreover, as we will see later, the effects due to the Lorentz boost of the three-quark system are crucial 1 .

Among the various possibilities of setting up a relativistic quantum the-

proceedings: submitted to World Scientific on November 24, 2018 
ory $\mathrm{O}$, here we choose the point-form formulation. It is characterized by several distinctive features. All the dynamics is contained in the four-momentum operators, which commute among themselves and can be simultaneously diagonalized. The generators of the Lorentz boosts contain no interactions and, therefore, are purely kinematic; the theory is thus manifestly covariant. In practice, the point-form formulation allows for a proper treatment of the Lorentz boosts of the three-quark wave functions and for an accurate calculation of the matrix elements of the electromagnetic current operator 6 . Due to the fact that the GBE CQM uses a relativistic kinetic energy operator, the full Hamiltonian leads to a mass operator fulfilling all the necessary commutation relations of the Poincaré group $\mathrm{Z}$, even if the quark-quark interaction consists of a phenomenological confinement and of an instantaneous GBE potential.

\section{The point-form approach to the scattering amplitude}

The starting point are the solutions of the eigenvalue problem for the Hamiltonian

$$
H=\sum_{i=1}^{3} \sqrt{\vec{k}_{i}^{2}+m_{i}^{2}}+\sum_{i<j=1}^{3}\left[V^{\operatorname{conf}}(i, j)+V^{\mathrm{GBE}}(i, j)\right],
$$

where $m_{i}$ are the masses and $\vec{k}_{i}$ the three-momenta of the constituent quarks in the nucleon rest frame, respectively. The confinement interaction $V^{\text {conf }}$ and the spin-spin component of the pseudoscalar GBE hyperfine interaction $V^{\mathrm{GBE}}$ produce a low-energy baryon spectrum in remarkable agreement with experimental results 3 .

The three-quark wave functions refer to constituent quarks in the nucleon rest frame, i.e. with total momentum $\vec{P}=0$. Therefore, they can be interpreted also as eigenstates of the mass operator including interactions 6

$$
M=\sqrt{P^{\mu} P_{\mu}}=M_{\text {free }}+M_{\text {int }},
$$

where $P^{\mu}=P_{\text {free }}^{\mu}+P_{\text {int }}^{\mu}$ is the four-momentum operator with inferactions inserted according to the Bakamjian-Thomas (BT) construction $\mathrm{G}$ in point form. The most general representation of such eigenstates is defined in the product space $\mathcal{H}_{1} \otimes \mathcal{H}_{2} \otimes \mathcal{H}_{3}$ of one-particle spin- $\frac{1}{2}$, positive-mass, positiveenergy representations of the Poincaré group

$$
|\psi\rangle \equiv\left|p_{1}, \lambda_{1}\right\rangle \otimes\left|p_{2}, \lambda_{2}\right\rangle \otimes\left|p_{3}, \lambda_{3}\right\rangle,
$$

where $p_{i}$ are the individual quark four-momenta and $\lambda_{i}$ the $z$-projections of their spins. A general Lorentz transformation $U_{\Lambda}$ on $|\psi\rangle$ produces three 
different Wigner rotations. It is more convenient to first introduce so-called velocity states 6 by applying a particular Lorentz boost $U_{B(v)}$ to the centerof-momentum states, which are defined analogously to Eq. (3) but fulfil the constraint $\vec{P}=\overrightarrow{k_{1}}+\overrightarrow{k_{2}}+\overrightarrow{k_{3}}=0$,

$$
\left|v ; \vec{k}_{1}, \vec{k}_{2}, \vec{k}_{3} ; \mu_{1}, \mu_{2}, \mu_{3}\right\rangle=U_{B(v)}\left|k_{1}, k_{2}, k_{3} ; \mu_{1}, \mu_{2}, \mu_{3}\right\rangle=\prod_{i=1}^{3} D_{\lambda_{i} \mu_{i}}^{1 / 2}\left[R_{W}\left(k_{i}, B(v)\right)\right]|\psi\rangle
$$

and $p_{i}=B(v) k_{i}$. Under a general Lorentz transformation $U_{\Lambda}$ and for canonical boosts, the velocity state (4) transforms as

$$
\begin{aligned}
& U_{\Lambda}\left|v ; \vec{k}_{1}, \vec{k}_{2}, \vec{k}_{3} ; \mu_{1}, \mu_{2}, \mu_{3}\right\rangle= \\
= & \prod_{i=1}^{3} D_{\mu_{i}^{\prime} \mu_{i}}^{1 / 2}\left(R_{W}\right)\left|\Lambda v ; R_{W} \vec{k}_{1}, R_{W} \vec{k}_{2}, R_{W} \vec{k}_{3} ; \mu_{1}^{\prime}, \mu_{2}^{\prime}, \mu_{3}^{\prime}\right\rangle ;
\end{aligned}
$$

the Wigner rotations $R_{W}$ are now all the same and the spins can thus be coupled together to a total spin as in nonrelativistic theory.

After setting up the proper Lorentz boosts of the nucleon state, the next task is to calculate the matrix element of the electromagnetic current operator. Following the formalism of Ref. 6 , it is possible to show that

$$
\begin{array}{r}
\left\langle\psi^{\prime}\left(P^{\prime}, \sigma^{\prime}\right)\left|J^{\mu}(0)\right| \psi(P, \sigma)\right\rangle=\int d^{4} Q B(Q)_{b}^{\mu}\left\langle\psi^{\prime}\left(P^{\prime}, \sigma^{\prime}\right)\left|J^{b}(Q)\right| \psi(P, \sigma)\right\rangle \\
=\int d^{4} Q \delta^{4}\left(P^{\prime}-P-Q\right) \sum_{b, r^{\prime}, r}\left(P \frac{1}{2} \sigma ; Q b r^{\prime} r \mid P^{\prime} \frac{1}{2} \sigma^{\prime}\right) \times F_{r^{\prime} r}^{b}\left(Q^{2}\right),
\end{array}
$$

where $b=0,1,2$ for $Q^{2}<0$ and $r, r^{\prime}= \pm \frac{1}{2},\left|r-r^{\prime}\right|=0, \pm 1 . \quad J^{b}$ is an irreducible tensor of the Poincaré group, ensuring that $J^{\mu}$ is covariant and conserved. Moreover, the Wigner-Eckhart theorem allows for the replacement of the matrix element of $J^{b}$ by a linear combination of products of ClebshGordan coefficients of the Poincaré group (linking a time-like $P$ and a spacelike $Q$ to a time-like $P^{\prime}$ ) and of reduced matrix elements $F_{r^{\prime} r}^{b}$, which can be identified with the invariant form factors. By choosing the convenient Breit frame with the 3 -momentum along the $\hat{z}$ axis, the initial and final velocities are given by $m_{N} v_{i}=P_{\mathrm{st}}=\left(\sqrt{m_{N}^{2}+(Q / 2)^{2}}, 0,0,-Q / 2\right)$ and $m_{N} v_{f}=P_{\mathrm{st}}^{\prime}=$ $\left(\sqrt{m_{N}^{2}+(Q / 2)^{2}}, 0,0, Q / 2\right)$, respectively, where $m_{N}$ denotes the nucleon mass.

Assuming the Impulse Approximation (IA), i.e. assuming that the virtual photon hits the quark labelled "1" leaving the "2", 3 " as spectators, the 
invariant form factors of Eq. (6) become 6.

$$
\begin{aligned}
& F_{r^{\prime} r}^{b}= 3 \int d \vec{k}_{2} d \vec{k}_{3} d \vec{k}_{2}^{\prime} d \vec{k}_{3}^{\prime} \psi_{r^{\prime}}^{*}\left(k_{2}^{\prime} \mu_{2}^{\prime}, k_{3}^{\prime} \mu_{3}^{\prime}\right) \psi_{r}\left(k_{2} \mu_{2}, k_{3} \mu_{3}\right) \\
& \delta^{3}\left[k_{2}^{\prime}-B^{-1}\left(v_{f}\right) B\left(v_{i}\right) k_{2}\right] \delta^{3}\left[k_{3}^{\prime}-B^{-1}\left(v_{f}\right) B\left(v_{i}\right) k_{3}\right] \\
& D_{\mu_{2}^{\prime} \mu_{2}}^{\frac{1}{2}}\left[R_{W}\left(k_{2}, B^{-1}\left(v_{f}\right) B\left(v_{i}\right)\right)\right] D_{\mu_{3}^{\prime} \mu_{3}}^{\frac{1}{2}}\left[R_{W}\left(k_{3}, B^{-1}\left(v_{f}\right) B\left(v_{i}\right)\right)\right] \\
& D_{\lambda_{1}^{\prime} \mu_{1}^{\prime}}^{\frac{1}{2} *}\left[R_{W}\left(k_{1}^{\prime}, B\left(v_{f}\right)\right)\right]\left\langle p_{1}^{\prime} \lambda_{1}^{\prime}\left|\left(\gamma^{b} f_{1}+i \frac{\sigma^{b \nu}\left(p_{1 \nu}^{\prime}-p_{1 \nu}\right)}{2 m_{1}} f_{2}\right)\right| p_{1} \lambda_{1}\right\rangle D_{\lambda_{1} \mu_{1}}^{\frac{1}{2}}\left[R_{W}\left(k_{1}, B\left(v_{i}\right)\right)\right]
\end{aligned}
$$

where the standard form for the relativistic single-particle current matrix element for quark label "1" has been used, that contains also the quark form factors $f_{1}\left(Q^{2}\right), f_{2}\left(Q^{2}\right)$. However, the following results have been obtained assuming point-like constituent quarks, i.e. $f_{1}\left(Q^{2}\right)=1$ and $f_{2}\left(Q^{2}\right)=0$

From Eq. (7) one obtains the nucleon Sachs form factors through 6

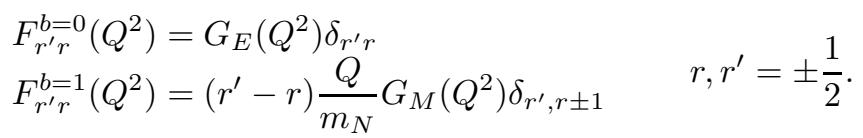

Note that only the $b=0$ and $b=1$ components of $F_{r^{\prime} r}^{b}$ are needed for the electric and magnetic form factors, respectively. There is no new information in $F_{r^{\prime} r}^{b=2}$. Therefore, the general result is recovered that for a spin- $\frac{1}{2}$ state only two independent form factors are needed. The structure of the Poincaré group allows for an easy generalization of this result in the point-form framework, where the matrix element of Eq. (7) for a spin- $j$ state is parametrized in terms of $2 j+1$ independent reduced matrix elements 6 .

\section{Results}

The predictions of the GBE CQM 1 for the nucleon electromagnetic form factors are shown by the solid line (PFSA) in Fig. 1. Their properties at zero momentum transfer are reflected by the charge radii and magnetic moments given in Table 1. The results were calculated along the lines explained in the previous Section. The input consists only of the proton and neutron threequark wave functions as produced by the solution of the eigenvalue problem for the Hamiltonian (11).

A very good description of both the proton and neutron electromagnetic structure is achieved, particularly at low momentum transfer. The dashed line represents the nonrelativistic IA result (NRIA), i.e. with the standard nonrelativistic form of the current operator and no Lorentz boosts applied to the nucleon wave functions. Evidently, relativity plays a major role here.

proceedings: submitted to World Scientific on November 24, 2018 

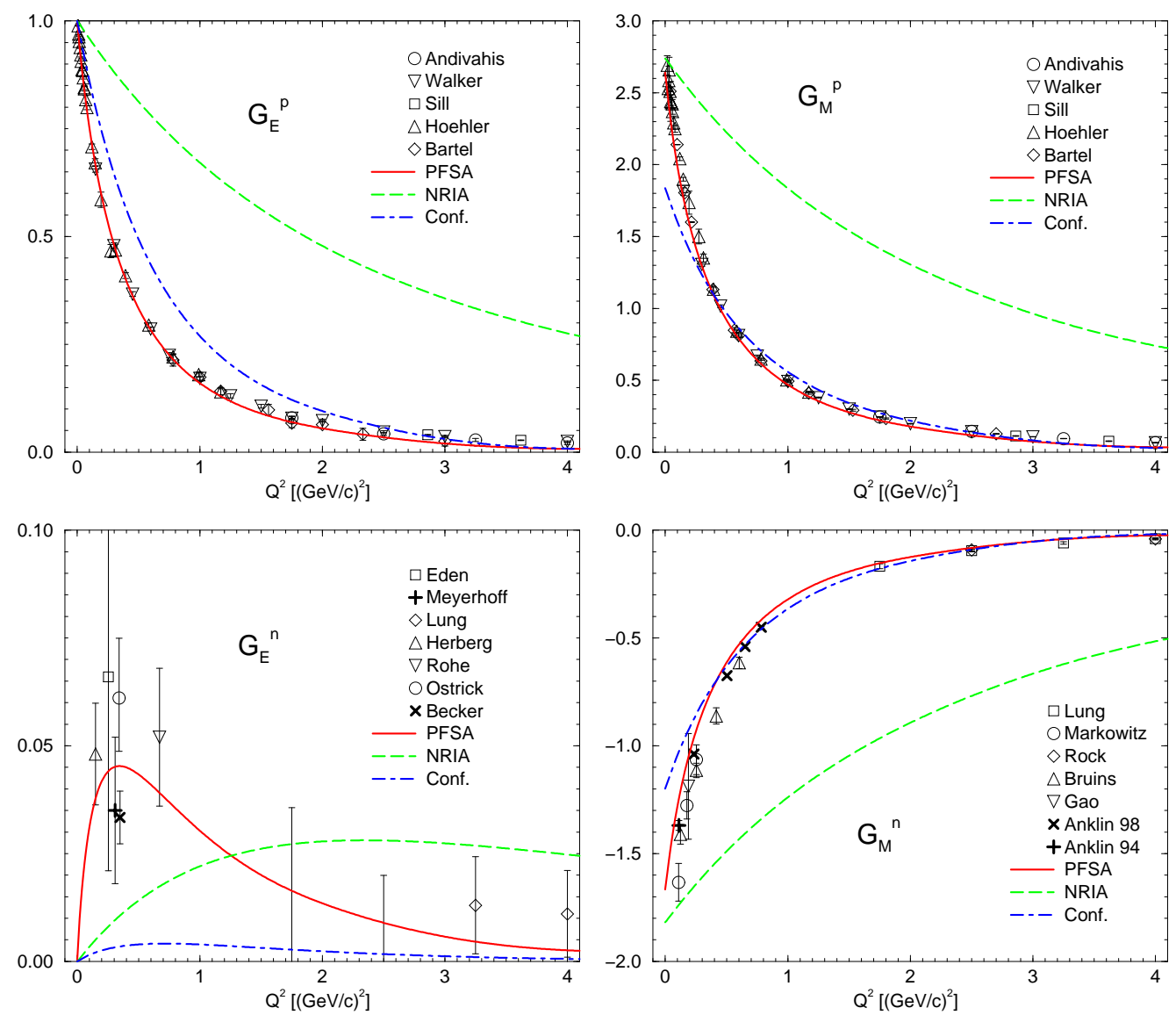

Figure 1. Proton (upper) and neutron (lower) electric (left) and magnetic (right) form factors as predicted by the GBE CQM $\mathrm{G}$ in IA (PFSA, solid lines). A comparison is given to the nonrelativistic gesults (NRIA, dashed) and the case with the confinement interaction only (dashed-dotted) 6 . The experimental data are from Ref. $\mathrm{B}$.

The importance of proper Lorentz boosts can be guessed by the $\delta$ functions acting on the spectator quarks in Eq. (7): $k_{i}^{\prime}=B^{-1}\left(v_{f}\right) B\left(v_{i}\right) k_{i} \quad i=2,3$. At low momentum transfer, this constraint becomes $k_{i}^{\prime} \simeq k_{i}-\frac{\omega_{i}}{m_{N}} Q$, with $\omega_{i}$ the energy of the $i$-th spectator quafk, and it has to be compared with the nonrelativistic result $k_{i}^{\prime}=k_{i}-\frac{1}{3} Q$ b. The expectation value of $\omega_{i}$ for the employed nucleon wave functions is $\left\langle\omega_{i}\right\rangle=768 \mathrm{MeV}$. Therefore, $\frac{\omega_{i}}{m_{N}}>\frac{1}{3}$, because even for low $Q^{2}$ the individual quark momenta are large. This result 
Table 7 . Proton and neutron charge radii and magnetic moments as predicted by the GBE CQM 2 in IA (PFSA). A comparison is given alpo to the nonrelativistic results (NRIA) and the case with the confinement interaction only $\mathrm{G}$.

\begin{tabular}{lrrrl}
\hline & PFSA & NRIA & Conf. & \multicolumn{2}{l}{ Experimental } \\
\hline$r_{p}^{2}\left[\mathrm{fm}^{2}\right]$ & 0.75 & 0.10 & 0.37 & $0.774(27)$ 10 $0.780(25)$ 11 \\
$r_{n}^{2}\left[\mathrm{fm}^{2}\right]$ & -0.12 & -0.01 & -0.01 & $-0.113(7)$ \\
$\mu_{p}[\mathrm{n} . \mathrm{m}]$. & 2.64 & 2.74 & 1.84 & $2.792847337(29) 13$ \\
$\mu_{n}[\mathrm{n} . \mathrm{m}]$. & -1.67 & -1.82 & -1.20 & $-1.91304270(5) \mathrm{13}$ \\
\hline
\end{tabular}

turns into a faster fall off of $G_{E}\left(Q^{2}\right)$ and a consequent bigger charge radius.

Noteworthy, the results here presented are obtained with point-like constituent quarks. At least for the range of momentum transfers considered in Fig. 11, there is no need to introduce constituent quark form factors (or any other phenomenological parameters heyond the CQM) 6 , at variance with other previous relativistic studies $14,15,16$. It is only with regard to the magnetic moments that there remains a small difference between the theoretical predictions and the experimental data. All this turns out in a underestimation of the dipole profile of $G_{M}\left(Q^{2}\right)$ and, consequently, in an overestimation of the recent CEBAF data for $\mu G_{E} / G_{M}$ for the proton 17 , as shown by the upper curve in Fig. 2. The lower curve is produced by the same calculation but replacing the correct experimental value of $\mu$ by the theoretical value $\mu=2.64$ consistent with the calculation of $G_{E}, G_{M}$ (see Table 11). It indicates that there is no violation of charge conservation (as it could wrongly appear from the upper curve for $Q^{2} \rightarrow 0$ ), rather it gives a "graphical" representation of the present discrepancy of calculations with experimental data for $G_{M}\left(Q^{2}\right)$.

In order to get an idea of the role of the GBE hyperfine interaction in the electromagnetic form factors calculations involving only the confinement potential have been performed $\mathrm{O}$ and are shown by the dot-dashed line in Fig. 1. All observables seem already in reasonable agreement, as well as charge radii and magnetic momenta. Only the charge structure of the neutron would remain much too small due to the absence of a mixed-symmetry component in the wave function. Though this breaking of SU(6) symmetry brought about by the hyperfine interaction is very small 3 , it plays a crucial role in reproducing the charge radius and $G_{E}\left(Q^{2}\right)$ for the neutron $\mathbf{A}$.

\section{Conclusions}

The theoretical predictions for elastic nucleon form factors obtained in the point-form approach and using the GBE interaction 2, are found to be in remarkably good agreement with all experimental data (charge radii, magnetic moments, electric and magnetic form factors) both for the proton and the neutron. No further ingredients beyond the quark model wave functions

proceedings: submitted to World Scientific on November 24, 2018 


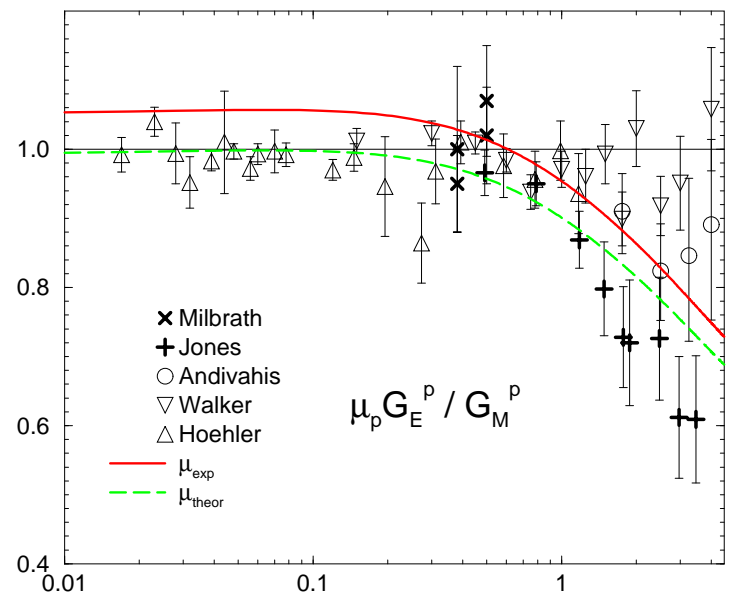

Figure 2. The $\mu G_{E} / G_{M}$ ratio for the proton. Upper curve obtained with experimental

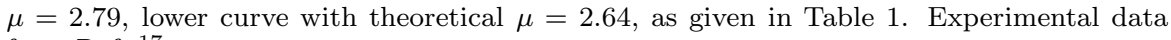
from Ref. 17 .

(such as constituent quark form factors etc.) have been employed. Only relativistic boost effects are properly included in point-form relativistic quantum mechanics 4 .

The matrix elements of the electromagnetic current operator have been computed in IA. Current conservation in Breit frame requires the $b=3$ component of the current operator to vanish identically. This is not true in IA $\mathbf{A l}$. However, Eq. (8) shows that physical observables are linked to components that are not affected by this approximation. Therefore, the effect of manybody current operators (needed to restore current conservation) should hopefully be small, and could be responsible for the small discrepancy observed in the values of magnetic momenta.

The present results have been obtained also assuming point-like constituent quarks. This suggests that these quasi-particles should be anyway small. It will be very interesting to explore this possibility, together with a necessary exploration of nucleon electromagnetic transitions to other resonances in a consistent fashion.

\section{Acknowledgments}

Numerous discussions with S. Boffi, L.Ya. Glozman, W. Klink, W. Plessas and R.F. Wagenbrunn are greatly acknowledged. 


\section{References}

1. L.Ya. Glozman and D.O. Riska, Phys. Rept. 268, 263 (1996).

2. L.Ya. Glozman, W. Plessas, K. Varga and R.F. Wagenbrunn, Phys. Rev. D 58, 094030 (1998).

3. L.Ya. Glozman, Z. Papp, W. Plessas, K. Varga and R.F. Wagenbrunn, Phys. Rev. C 57, 3406 (1998).

4. R.F. Wagenbrunn, S. Boffi, W. Klink, W. Plessas and M. Radici, nucl-th/0010048.

5. P.A.M. Dirac, Rev. Mod. Phys. 21, 392 (1949).

6. W.H. Klink, Phys. Rev. C 58, 3587 (1998).

7. B.D. Keister and W.N. Polyzou, Adv. Nucl. Phys. 20, 225 (1991).

8. B. Bakamjian and L.H. Thomas, Phys. Rev. 92, 1300 (1953).

9. L. Andivahis et al., Phys. Rev. D 50, 5491 (1994); R.C. Walker et al., Phys. Rev. D 49, 5671 (1994); A.F. Sill et al., Phys. Rev. D 48, 29 (1993); G. Höhler et al., Nucl. Phys. B 114, 505 (1976); W. Bartel et al., Nucl. Phys. B 58, 429 (1973); T. Eden et al., Phys. Rev. C 50, R1749 (1994); M. Meyerhoff et al., Phys. Lett. B 327, 201 (1994); A. Lung et al., Phys. Rev. Lett. 70, 718 (1993); C. Herberg et al., Eur. Phys. J. A 5, 131 (1999); D. Rohe et al., Phys. Rev. Lett. 83, 4257 (1999); M. Ostrick et al., Phys. Rev. Lett. 83, 276 (1999); J. Becker et al., Eur. Phys. J. A 6, 329 (1999); P. Markowitz et al., Phys. Rev. C 48, R5 (1993); S. Rock et al., Phys. Rev. Lett. 49, 1139 (1982); E.E.W. Bruins et al., Phys. Rev. Lett. 75, 21 (1995); H. Gao et al., Phys. Rev. C 50, R546 (1994); H. Anklin et al., Phys. Lett. B 428, 248 (1998); H. Anklin et al., Phys. Lett. B 336, 313 (1994).

10. R. Rosenfelder, Phys. Lett. B 479, 381 (2000).

11. K. Melnikov and T. van Ritbergen, Phys. Rev. Lett. 84, 1673 (2000).

12. S. Kopecky, P. Riehs, J.A. Harvey and N.W. Hill, Phys. Rev. Lett. 74, 2427 (1995).

13. D.E. Groom et al., Eur. Phys. J. C 15, 1 (2000).

14. F. Cardarelli, E. Pace, G. Salmè and S. Simula, Phys. Lett. B 357, 267 (1995); Few-Body Syst. Suppl. 11, 66 (1999); F. Cardarelli and S. Simula, Phys. Lett. B 467, 1 (1999); nucl-th/0006023.

15. A. Szczepaniak, C.R. Ji and S.R. Cotanch, Phys. Rev. C 52, 2738 (1995).

16. F. Coester and D.O. Riska, Few-Body Syst. 25, 29 (1998).

17. M.K. Jones et al., Phys. Rev. Lett. 84, 1398 (2000).

proceedings: submitted to World Scientific on November 24, 2018 